\title{
A Piezoelectric Energy Harvester Based on Pressure Fluctuations in Kármán Vortex Street
}

\author{
Dung-An Wang ${ }^{1, *}$, Huy-Tuan Pham ${ }^{1}$, Chia-Wei Chao ${ }^{1}$, Jerry M. Chen ${ }^{2}$ \\ ${ }^{1}$ Graduate Institute of Precision Engineering, National Chung Hsing University, Taichung 40227, Taiwan, \\ $R O C$ \\ 2 Department of Mechanical Engineering, National Chung Hsing University, Taichung 40227, Taiwan, ROC \\ * Corresponding author. Tel: +886 422840531, Fax: +886 422858362, E-mail: daw@dragon.nchu.edu.tw
}

\begin{abstract}
We have developed a new energy harvester for harnessing energy from the Kármán vortex street behind a bluff body in a water flow. It converts flow energy into electrical energy through oscillation of a piezoelectric film. Oscillation of the piezoelectric film is induced by pressure fluctuation in the Kármán vortex street. Prototypes of the energy harvester are fabricated and tested. Experimental results show that an open circuit output voltage of $0.12 \mathrm{~V}_{\mathrm{pp}}$ and an instantaneous output power of $0.7 \mathrm{nW}$ are generated when the pressure oscillates with an amplitude of $\sim 0.3 \mathrm{kPa}$ and a frequency of $\sim 52 \mathrm{~Hz}$. This approach has the potential of converting hydraulic energy into electricity for powering wireless devices. The low output power of the device can be improved by an optimization design procedure or by adopting a piezoelectric material with higher piezoelectric constants. An array of these devices with multiple resonant frequencies may be considered for energy harvesting from ambient flow sources.
\end{abstract}

Keywords: Energy Harvester, Piezoelectric, Kármán vortex street

\section{Introduction}

Recent development of wireless sensor networks allowing real-time industrial process monitoring, machine health monitoring, environment monitoring, healthcare applications, and traffic control demands an economical source of energy not requiring fuel or replacement of finite power stores. Considerable effort is focused on use of renewable energy coming from natural resources such as flowing water, rain, tides, wind, sunlight, geothermal heat and biomass. R enewable energy from small-scale hydro, modern biomass, wind, solar, geothermal and biofuels accounted for $2.7 \%$ of global final energy consumption in 2008 and is growing very rapidly [1].

Small hydro systems using turbines/wheels can be used to convert mechanical energy from water flow into electricity. Krähenbühl et al. [2] designed an electromagnetic harvester based on a turbine driven by water pressure drop in throttling valves and turbo expanders in plants that outputs $150 \mathrm{~W}$ power with a rotation speed of $490000 \mathrm{rpm}$. Their device comprises a turbine and a permanent generator. A detailed electromagnetic machine design, rotor dynamics analysis and a thermal design were required to construct their energy harvester. Holmes et al. [3] reported an electromagnetic generator integrated with a microfabricated axial-flow microturbine. The power output of the fabricated microdevice can be as high as $1.1 \mathrm{~mW}$ per stator when operated at a rotation speed of $30000 \mathrm{r} \mathrm{pm}$, but the fabrication processes for their prototype involve deep reactive ion etching, multilevel electroplating, SU8 processing and laser micromachining. Herrault et al. [4] presented a rotary electromagnetic generator to harvest the mechanical energy of an air-driven turbine. The fabrication of their device requires electroforming, magnet demagnetization and laser machining. A maximum output power of $6.6 \mathrm{~mW}$ is attained when their device is driven at a rotation speed of 392000 rpm by an air turbine. The devices of Krähenbühl et al. [2], Holmes et al. [3] and Herrault et al. [4] require elaborate techniques for fabrication of their stator-rotor subcomponents and high rotation speeds for efficient energy harvesting. A device with simpler structure design 
and ease of application may be needed to extract energy from fluid motion for microsensor and communications applications.

Installations with miniaturized pipe-line systems may provide an alterntive for harvesting small scale water flow energy. Sanchez-Sanz et al. [5] assessed the feasibility of using the unsteady forces generated by the Kármán street around a micro-prism in the laminar flow regime for energy harvesting. They presented design guidelines for their devices, but fabrication and demonstration of the proposed device are not shown in their work. Allen and Smits [6] used a piezoelectric membrane placed behind the Kármán vortex street formed behind a bluff body to harvest energy from fluid motion. They examined the response of the membrane to vortex shedding. The power output of the membrane is not presented. Taylor et al. [7] developed an eel structure of piezoelectric polymer to convert mechanical flow energy to electrical power. They have focused on characterization and optimization of the individual subsystems of the eel system with a generation and storage units in a wave tank. Design and deployment of the eel system need further investigation. Tang et al. [8] designed a fluttermill to generate electricity by extracting energy from fluid flow. Their structure is similar to the eel systems of Allen and Smits [6] and Taylor et al. [7]. They investigate the energy transfer between the structure and the fluid flow through an analytical approach. These authors utilized the flow-induced vibrations of fluid-structure interaction system to extract energy from the surrounding fluid flow [9]. The eel structures of Allen and Smits [6], Taylor et al. [7] and Tang et al. [8] have the potential to generate power from milli-watts to many watts depending on system size and flow velocity, but a power-generating eel has not been demonstrated.

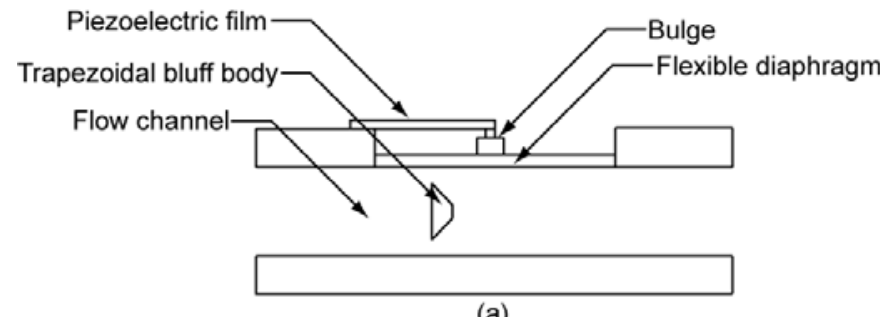

(a)

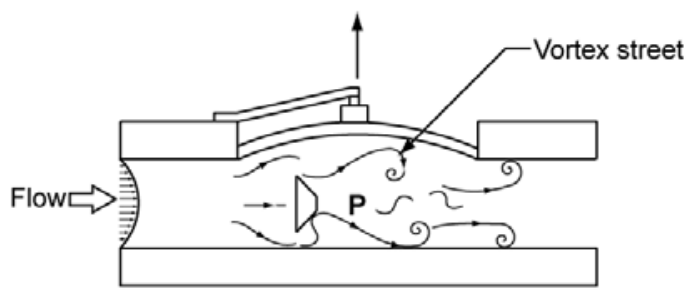

(b)

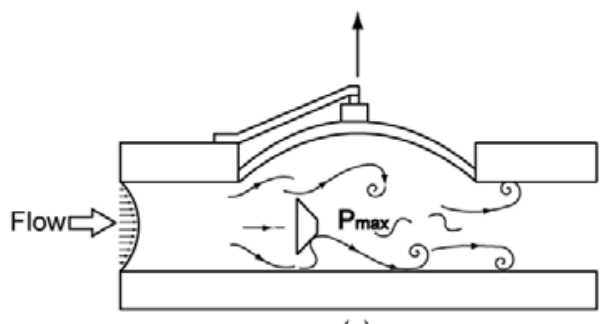

(c)

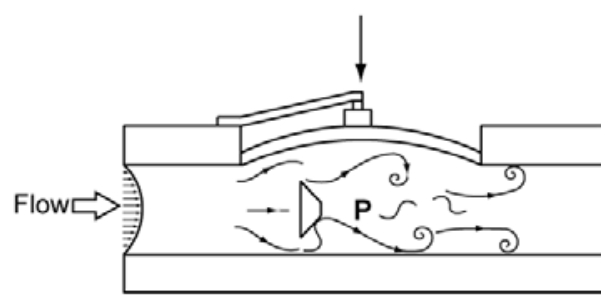

(d)

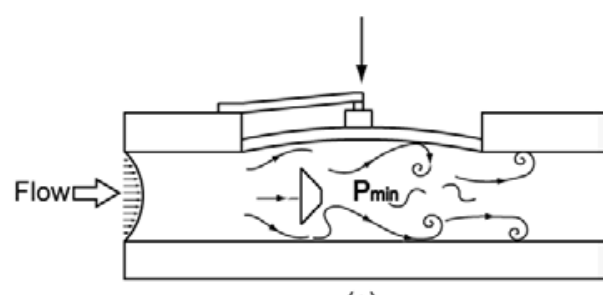

(e)

Fig. 1. Operation of a piezoelectric energy harvester.

In this report, we demonstrate a new device for energy harvesting from pressure fluctuations in the Kármán vortex street, where a p iezoelectric film is placed on top of a flexible 
diaphragm, which is located in the wake of a bluff body. The piezoelectric film oscillates on a flexible diaphragm due to the vortices shed from the bluff body in a water flow. As illustrated in Fig. 1(a), a flow channel with a flexible diaphragm is connected to a flow source. A piezoelectric film of a cantilever type is glued to a bulge affixed to the top surface of the flexible diaphragm. A bluff body is placed in the flow channel. Pressure in the flow channel behind the bluff body may fluctuate with the same frequency as the pressure variation caused by the Kármán vortex street. Fig. 1(b) shows that the pressure in the channel causes the diaphragm and the piezoelectric film to deflect in the upward direction. As the pressure increases to the maximum, the diaphragm reaches its highest position (Fig. 1(c)). When the pressure drops, the diaphragm and the piezoelectric film deflect downward (Fig. 1(d)). As the pressure decreases to the minimum, the diaphragm reaches its lowest position (Fig. 1(e)). Thus, by placing the energy harvester in a flow source, the oscillating movement of the diaphragm with the cantilever piezoelectric film attached to it makes the energy harvesting possible.
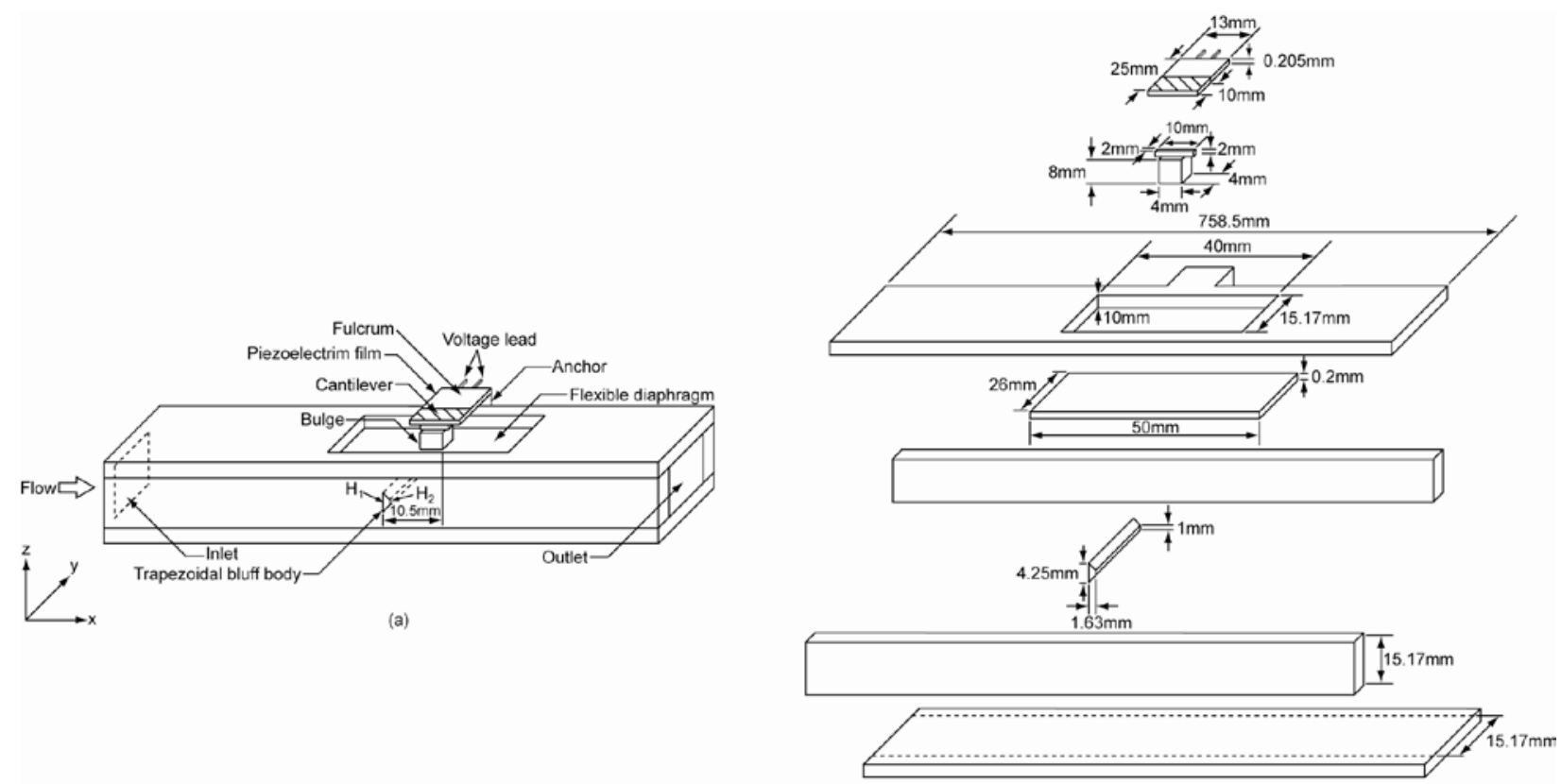

Fig. 2. (a) An assembled energy harvester. (b) Components of the energy harvester..

\section{Methodology}

\subsection{Operational principle}

A piezoelectric energy harvester considered in this investigation is shown in Fig. 2(a). Fig. 2(b) is an exploded view of the energy harvester. The dimensions of the energy harvester are indicated in Fig. 2(b). It consists of a flow channel, a bluff body, a polydimethylsiloxane (PDMS) diaphragm bonded to the channel, and a piezoelectric film attached to the PDMS diaphragm through a bulge made of acrylic blocks. Flow past a bluff body creates an unstable wake in the form of alternating vortices and induces periodic pressure variation [10]. The frequency at which the vortices are shed from the bluff body is given by the Strouhal number, St, $\mathrm{S} t=\omega \ell / U_{\infty}$ [11], where $\omega$ is the frequency of oscillating flow, $\ell$ is the characteristic length, and $U_{\infty}$ is the free-stream velocity. Vortex shedding from a circular cylinder immersed in a steady flow occurs in the range $1 \hat{0}<\mathrm{R} \& 1 \delta$, where $\mathrm{Re}$ is the Reynolds number, with an average Strouhal number $\omega d / 2 \pi U_{\infty} \approx 0.2$ [12]. For a trapezoidal bluff 
body, the Strouhal number is given as $\mathrm{S} \Leftarrow \omega H_{1} / U_{\infty} \quad$ [13], where $H_{1}$ is the height of the front side of the trapezoidal cylinder. T he front height $H_{1}$ and rear height $H_{2}$ of the trapezoidal cylinder are denoted in Fig. 2(a).

The piezoelectric film (LDT0-028K/L, Measurement Specialties, Inc., US) is a laminate including a polyvinylidene fluoride (PVDF) film, two silver electrode layers and a polyester (PE) layer. The PDMS flexible diaphragm has a thickness of $200 \mu \mathrm{m}$. The electrode layers with a thickness of $28 \mu \mathrm{m}$ are attached to the top and bottom surfaces of the PVDF film of 24 $\mu \mathrm{m}$. A $125^{\mu \mathrm{m}}$ PE layer is laminated to the top surface of the top electrode layer. The values of $d_{31}$ and $d_{33}$ of the PVDF are 23 and $-33 \mathrm{pm} \cdot V^{-1}$, respectively. The capacitance of the PVDF film is $380 \mathrm{pF} \cdot \mathrm{cm}^{-2}$. The Young's modulus and Poisson's ratio of the PVDF are 3 GPa and 0.35 , respectively. When used in a bending mode, the laminated piezoelectric film develops much higher voltage output when flexed than a non-laminated film. The neutral axis is in the PE layer instead of in the PVDF film so the film is strained more when flexed.

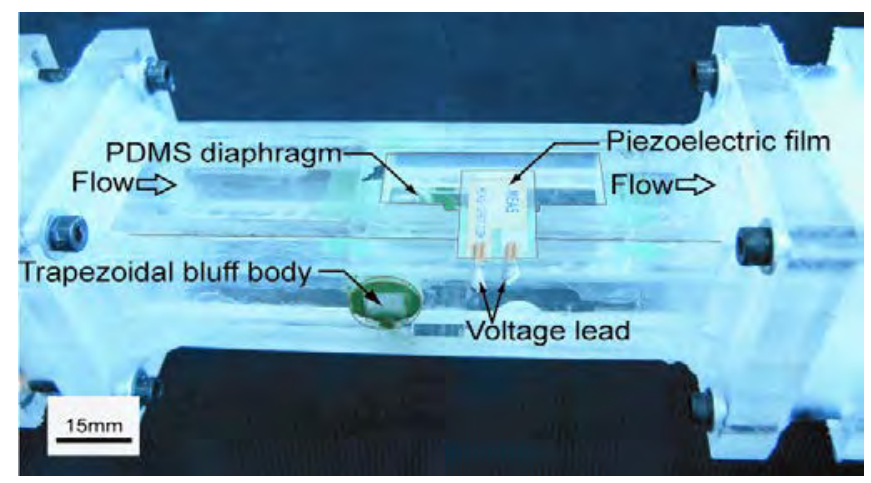

Fig. 3. Assembled energy harvester.

\subsection{Fabrication and Experiments}

In order to verify the effectiveness of the proposed energy harvesting device, prototypes of the energy harvester were fabricated. Fig. 3 is a photo of an assembled energy harvester. Its dimensions are indicated in Fig. 2(b). Fig. 4 is a photo of the experimental apparatus for testing of the fabricated device. The energy harvester is placed on an optical table for vibration isolation. From the bottom of a storage tank, an inlet pipe is run down to the inlet of the energy harvester. The water level in the storage tank is kept constant for a steady water flow at the inlet of the flow channel. Using gravity, water is forced into the inlet of the energy harvester. T ap water is pumped into the storage tank through a pump located in a recycle tank. An outlet pipe extending between the outlet of the energy harvester and the recycle tank provides a continuous supply of water. $\mathrm{T}$ he oscillating deflection of the piezoelectric film is measured by a fiberoptic displacement sensor (MTI-2000, MTI Instruments Inc., US). The generated voltage of the piezoelectric film is recorded and analyzed by a data acquisition unit (PCI-5114, National Instruments Co., US). The pressure in the pressure chamber is measured with a subminiature pressure sensor (PS-05KC, Kyowa Electronic Instruments Co. Ltd., Japan) embedded in the bottom plate of the flow channel. The pressure sensor is connected to a data acquisition unit (DBU-120A, Kyowa Electronic Instruments Co. Ltd., Japan). 


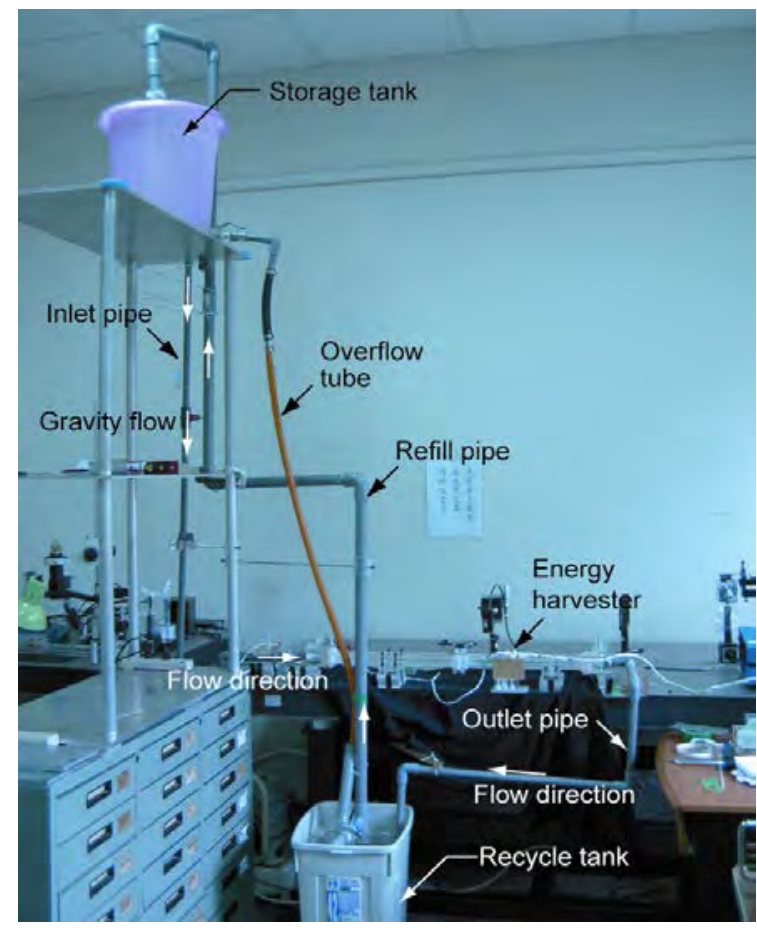

Fig. 4. A photo of the experimental setup.

\section{Results}

The experimental results are shown in Fig. 5. Fig. 5(a) shows the pressure history in the flow channel behind the bluff body, where the pressure oscillates with an amplitude of nearly 0.3 $\mathrm{kPa}$ and a frequency of $52 \mathrm{~Hz}$. The measured deflection history of the free end of the piezoelectric film is shown in Fig. 5(b). The film oscillates with an amplitude of about 20 $\mu \mathrm{m}$. The measured open circuit voltage generated by the piezoelectric film is shown in Fig. 5(c). The output peak-to-peak voltage is nearly $120 \mathrm{mV}_{\mathrm{pp}}$. The results shown in Figs. 5(a-c) are typical of those obtained over 20 s econds of measurements. Figs. 5(d-f) are the power spectral density corresponding to Figs. 5(a-c), respectively, but are based on all 20 seconds of measurements. Fast Fourier transform is used to compute the power spectral density. It can be seen from Figs. 5(d-f) that there is one obvious peak value of $52 \mathrm{~Hz}$, caused by the pressure fluctuation in the flow channel. The low-frequency noise, below $20 \mathrm{~Hz}$, observed in Figs. 5(d-f) can be attributed to the fact that flows in the experimental setup are always contaminated by ambient noise sources, and the geometry of the bluff body and the walls of the flow channel are not perfectly symmetric and smooth.

The average free-stream velocity $U_{\infty}$ measured in the experiments is $1.083 \mathrm{~m} / \mathrm{sec}$. T he Reynolds number Re of the flow can be determined by $\mathrm{R} \in \rho U_{\infty} D_{h} / \mu$, where $\mu$ is the dynamic viscosity of the water, $1.00 \approx 10^{-3} \mathrm{~Pa} \cdot \mathrm{sec}$, and $D_{h}$ is the hydraulic diameter, which is $15.17 \mathrm{~mm}$ for the flow channel considered. The calculated $\mathrm{Re}$ is $1.64 \times 10^{4}$, which is turbulent. The frequency of the pressure fluctuation is nearly $52 \mathrm{~Hz}$ (Fig. 5(d)), which is the rate at which the vortices are shed from the bluff body. Using the equation $\mathrm{S} \neq \omega H_{1} / U_{\infty}$, the corresponding value of $\mathrm{St}$ is estimated as 0.204. White [12] reported an average St about 0.21 for the shedding from a circular cylinder immersed in a steady flow occurring in the range $1 \hat{0}<\mathrm{R} \& 1 \delta$. The estimated value of St for a trapezoidal bluff body of the experiments is reasonable compared with the value 0.21 reported by White [12]. The experimental values of 
St for trapezoidal cylinders with the height ratio, $H_{2} / H_{1}$, ranging from 0.3 to 1.0 and the Re values of 100,150, and 200, have a range from approximately 0.135 to approximately 0.155 [13]. Based on the work of Norberg [14] for a circular cylinder, the variations in St are within the limit $0.19 \pm 0.01$ for the Re ranging from 250 to $3 \times 10^{5}$. Ho and Huerre [15] commented that the values of St changes from 0.016 for a laminar flow to $0.022-0.024$ for turbulent flow. Based on the reported experimental data [12-15], the estimated value of St, 0.204 , based on the experiments carried out in this investigation could be acceptable.

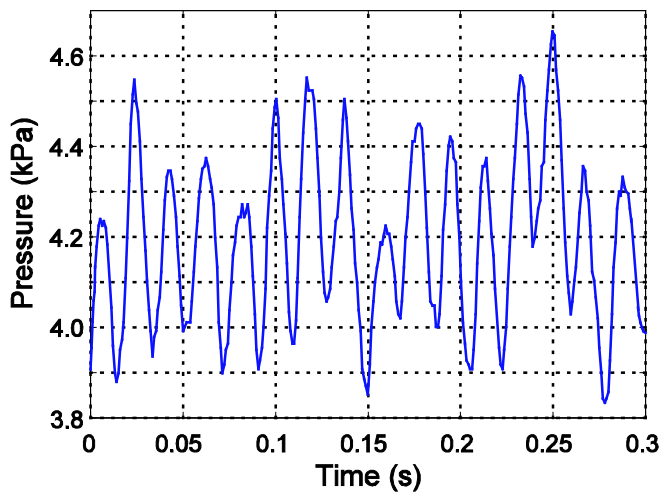

(a)

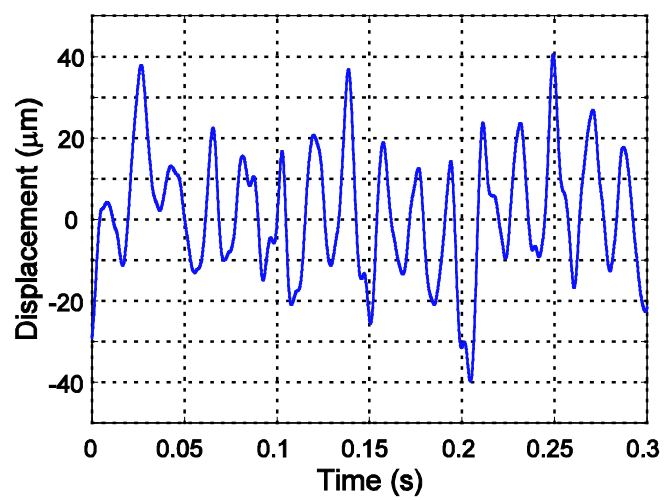

(b)

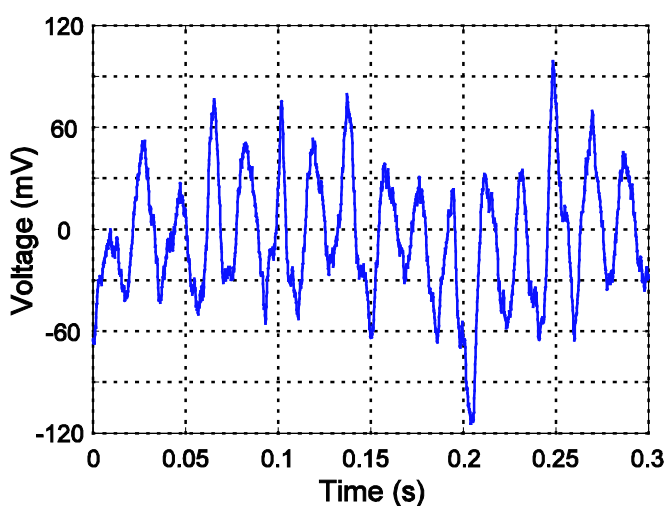

(c)

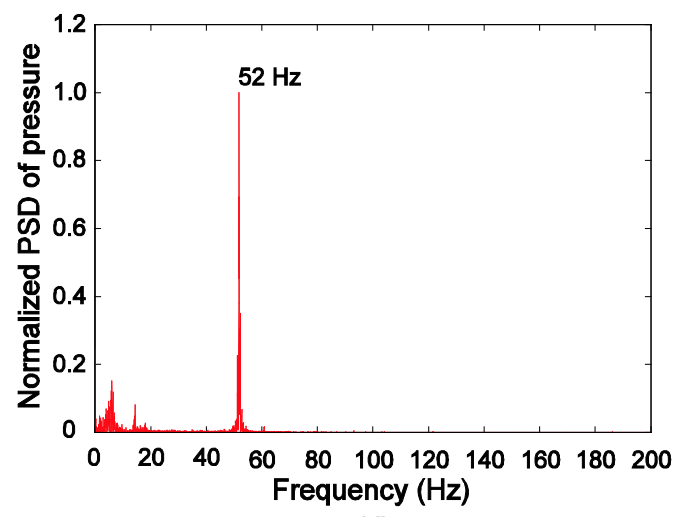

(d)

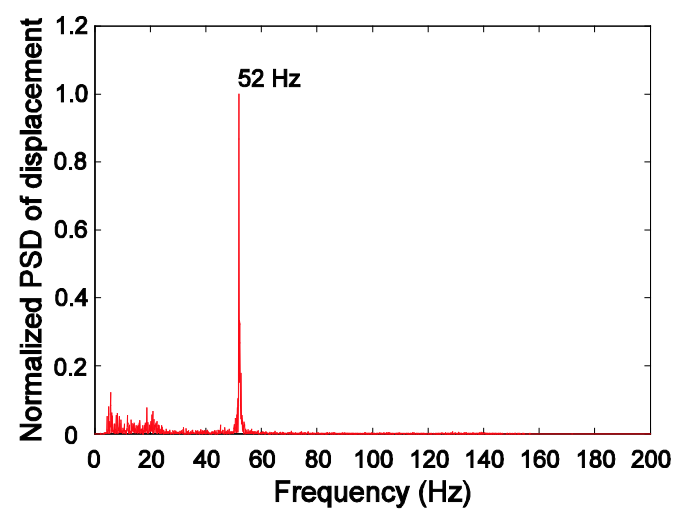

(e)

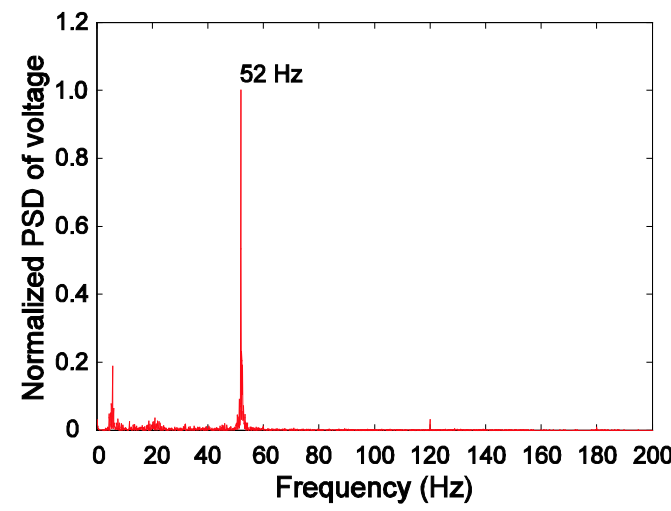

(f)

Fig. 5. Experimental results. (a) Pressure variation in the pressure chamber. (b) Deflection of the free end of the cantilever piezoelectric film. (c) Output voltage of the piezoelectric film. (d-f) Power spectral density corresponding to $(a-c)$.

A matched load can be connected to the device to evaluate the power output of the device. The internal electrical resistance of the device is measured by a LCR meter (WK 4235, 
Wayne Kerr Electronics, Ltd., UK). The instantaneous power can be expressed as $P=(\sqrt{2} \widetilde{V})^{2} / R$, where $R$ is the resistance value of the matched load and $\widetilde{V}$ is the root-meansquare value of the voltage drop across the matched load. By connecting the matched load of $655 \mathrm{k} \Omega$ to the device and detecting the voltage drop across the matched load, $14.89 \mathrm{~m} V_{r m s}$, the instantaneous power is determined as $0.7 \mathrm{nW}$.

\section{Discussions}

The output power of the device is relatively low, given the structure design of the flow channel, the bluff body and the cantilever piezoelectric film. In order to obtain a higher output power of the piezoelectric energy harvester, the dimensions and structure of the device can be optimized, and a piezoelectric material with higher piezoelectric constants can be adopted. In this investigation, the device is not operated at its resonance frequency. Most energy harvesting device based on piezoelectric effects have focused on single-frequency ambient energy, i.e. resonance-based energy harvesting [16]. The resonance frequency of the energy harvesting device can be tailored to the shedding frequency of the Kármán vortex street in order to increase the output power of the device. For random and broadband ambient flow sources, such a device may not be robust. A structure with multiple resonant frequencies may also be considered for energy harvesting from random vibrations with multiple resonant peaks, for example a segmented composite beam with embedded piezoelectric layers [17].

In order to generate the pressure fluctuation of the Kármán vortex street in the channel, a flow source assisted by gravity is used to force tap water into the flow channel in the laboratory environment. Energy can be harvested from pipe flows, blood flow in arteries $[18,19]$, or air flow in tire cavities. The proposed device can be deployed on slopes to provide electricity for wireless sensor networks for detection of landslides. Landslides are usually preceded by heavy rainfalls, and the device can harvest the energy of the water flow along slopes due to rainfall. E nergy harvesting from regular, periodic shedding can be integrated into tire pressure monitoring systems to harness the energy of air flow in tire cavities, or self-powering implantable and wireless devices in human bodies to convert the hydraulic energy of flow of body fluid.

\section{References}

[1] REN21, Renewables 2010 Global Status Report, 2010, pp. 15-16.

[2] D. Krähenbühl, C. Zwyssig, H. Weser, J.W. Kolar, Theoretical and experimental results of a mesoscale electric power generation system from pressurized gas flow, Journal of Micromechanics and Microengineering 19, 2009, 094009.

[3] A.S. Holmes, G. Hong, K.P. Pullen, Axial-flux permanent magnet machines for micropower generation, Journal of Microelectromechanical Systems 14, 2005, pp. 54-62.

[4] F. Herrault, C.-H. Ji, M. G. Allen, Ultraminiaturized high-speed permanent-magnet generators for milliwatt-level power generation, Journal of Microelectromechanical Systems 17, 2008, pp. 1376-1387.

[5] M. Sanchez-Sanz, B. Fernandez, A. Velazquez, Energy-harvesting microresonator based on the forces generated by the Kammon street around a rectangular prism, Journal of Microelectromechanical Systems 18, 2009, pp. 449-457.

[6] J.J. Allen, A.J. Smits, Energy harvesting eel, Journal of Fluid and Structures 15, 2001, pp. 629-640. 
[7] G.W. Taylor, J.R. Burns, S.M. Kammann, W. B. Powers and T. R. Welsh, The energy harvesting eel: A small subsurface ocean/river power generator, IEEE Journal of Oceanic Engineering 26, 2001, pp. 539-547.

[8] L. Tang, M.P. Païdoussis, J. Jiang, Cantilevered flexible plates in axial flow: Energy transfer and the concept of flutter-mill, Journal of Sound and Vibration 326, 2009, pp. 263-276.

[9] R.D. Blevins, Flow-induced vibration, Van Nostrand Reinhold, ed. 2, 1990.

[10]R. Violette, E. de Langre, J. Szydlowski, Computation of vortex-induced vibrations of long structures using a wake oscillator model: Comparison with DNS and experiments, Computers and Structures 85, 2007, pp. 1134-1141.

[11]D.F. Young, B.R. Munson, T.H. Okiishi, A brief introduction to fluid mechanics, John Wiley \& Sons, 2001.

[12]F.M. White, Fluid Mechanics, McGraw-Hill, 1986.

[13] Y.J. Chung, S.-H. Kang, Laminar vortex shedding from a trapezoidal cylinder with different height ratios, Physics of Fluids 12, 2000, pp. 1251-1254.

[14]C. Norberg, An experimental investigation of the flow around a circular cylinder: influence of aspect ratio, Journal of Fluid Mechanics 258, 1994, pp. 287-316.

[15] C.M. Ho, P. Huerre, Perturbed free shear layers, Annual Review of Fluid Mechanics 16, 1984, pp. 365-422.

[16] S. Adhikari, M.I. Friswell, D.J. Inman, Piezoelectric energy harvesting from broadband random vibrations, Smart Materials and Structures 18, 2009, 115005.

[17] S. Lee, B.D. Youn, B.C. Jung, Robust segment-type energy harvester and its application to a wireless sensor, Smart Materials and Structures 18, 2009, 095021.

[18]C. Mo, L.J. Radziemski, W.W. Clark, Experimental validation of energy harvesting performance for pressure-loaded piezoelectric circular diaphragms, Smart Materials and Structures 19, 2010, 075010.

[19]Z.L. Wang, J. Song, Piezoelectric nanogenerators based on zinc oxide nanowire arrays, Science 312, 2006, pp. 242-246. 